\title{
PREMIÈRES MESURES PHOTOMÉTRIQUES: DE LÉONARD DE VINCI À PIERRE BOUGUER
}

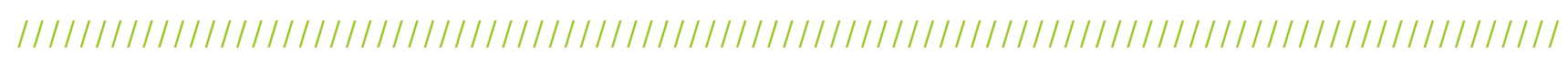

\section{Lionel SIMONOT}

Université de Poitiers, Institut Pprime, UPR CNRS 3346, Chasseneuil Futuroscope, France

lionel.simonot@univ-poitiers.fr

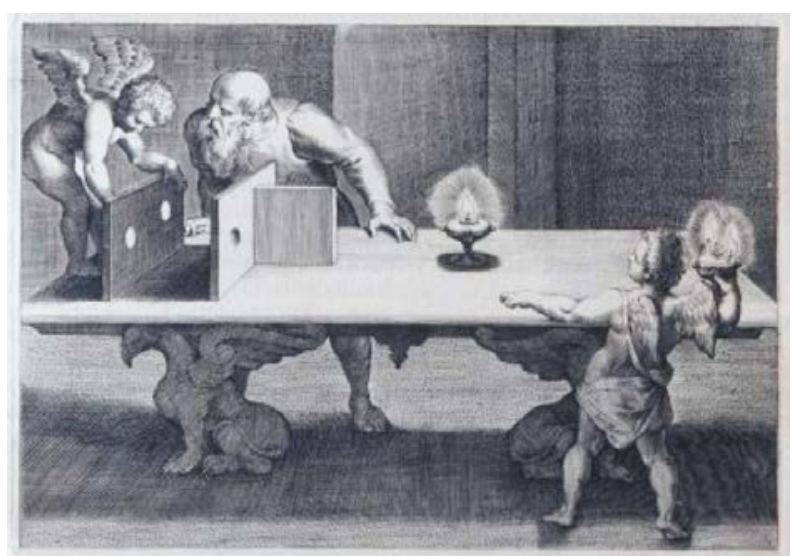

ui inventa le premier photomètre ? De Léonard de Vinci à Pierre Bouguer, il fallut plus de deux siècles pour établir le principe de mesures photométriques par égalisation visuelle entre les éclairements produits par la source de lumière considérée et par une source de référence. Après un essor important au XIX ${ }^{\mathrm{e}}$ pour caractériser les nouveaux dispositifs d'éclairage au gaz, les photomètres vont disparaitre au $\mathrm{XX}^{\mathrm{e}}$ siècle avec l'apparition des capteurs photoélectriques.

La photométrie est une discipline cousine de l'optique géométrique. Or, si cette dernière trouve ses racines dès le $\mathrm{III}^{\mathrm{e}}$ siècle avant J.C. avec les travaux d'Euclide, il faut attendre de longs siècles pour qu'émerge l'étude de la mesure quantitative de la lumière. Comment en effet mesurer la lumière de manière fiable ? Certes, le système

Comment mesurer une quantité de lumière ? Cette question fondamentale pour toute expérience optique n'allait pas de soi jusqu'au XVIII' siècle. Il fallait inventer un instrument et un protocole de mesure utilisant le seul photodétecteur alors disponible: l'œil humain. Plusieurs savants et quelques grands peintres contribuèrent sur plus de deux siècles à l'élaboration du premier photomètre.

https://doi.org/10.1051/photon/202010326

Article publié en accès libre sous les conditions définies par la licence Creative Commons Attribution License CC-BY (https://creativecommons.org/licenses/by/4.0), qui autorise sans restrictions l'utilisation, la diffusion, et la reproduction sur quelque support que ce soit, sous réserve de citation correcte de la publication originale.

visuel humain est particulièrement performant par sa capacité à détecter des luminosités allant de la quasi obscurité jusqu’à léblouissement, performance encore inégalée par les photodétecteurs actuels. Mais l'œil humain ne peut faire une mesure absolue de la lumière reçue d'autant qu'il s'adapte à la luminosité ambiante. Pour s'affranchir de ce défaut, il fallait inventer un " photomètre », un dispositif permettant de quantifier la luminosité d'une source de lumière par un observateur humain. Le principe repose sur l'égalisation visuelle entre les éclairements produits par la source à étudier et par une source de référence.

\section{L'INVENTION D'UN PEINTRE ?}

L'usage de la perspective et de la peinture à l'huile a donné une nouvelle liberté aux peintres de la Renaissance leur permettant de traduire avec un réalisme rarement atteint jusqu'alors les volumes et les reliefs. Quelques décennies plus tard, Léonard de Vinci a bénéficié de cette révolution technique et picturale, et a porté jusqu'à l'incarner, la maîtrise de la construction des ombres et des lumières. Ainsi, comme pour de nombreuses autres inventions, il n'est pas illégitime d'attribuer à Léonard de Vinci celle du premier photomètre. On trouve effectivement quelques schémas rudimentaires dans un de ses fameux codex (en l'occurrence le Manuscrit $C$ de France). Le principe de comparaison des ombres créées par deux sources y est décrit (figure 1). En modifiant la distance d'une des sources, il est possible d'égaliser la «force » des ombres. Le principe du photomètre est esquissé. Mais, en séquipant d'un miroir, vous pourrez constater dans le texte qui accompagne le schéma, que Léonard de Vinci propose une loi de décroissance de l'éclairement en inverse de $\bullet \bullet$ 


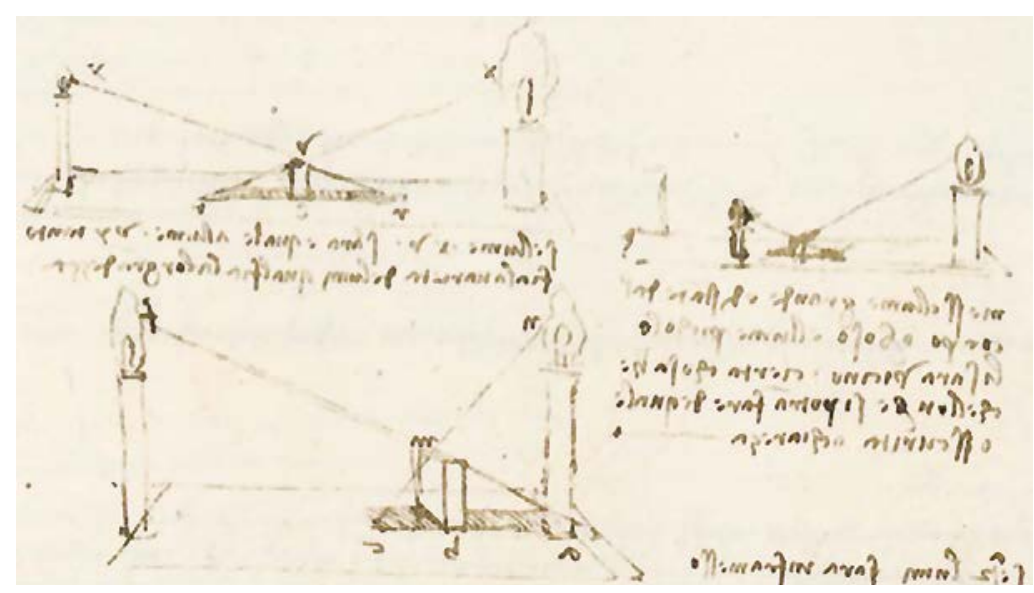

la distance, ce qui, nous le verrons par la suite, n'est pas correcte. Une telle erreur indique que le peintre n'a probablement pas mené des expérimentations très approfondies.

Plus d'un siècle plus tard, c'est un autre peintre qui semble illustrer le fonctionnement d'un photomètre. La gravure, présentée en tête d'article, est de Paul Rubens. Etonnant de voir une œuvre du peintre de la couleur en noir et blanc ! Il s'agit d'une illustration pour un traité d'optique en six livres publié en 1613. Son auteur, François d'Aguillon, est un savant jésuite. Son lien avec le peintre est plus évident par la classification des couleurs qu'il expose également dans son traité. Il propose, sans doute pour la première fois, d'ordonner les couleurs autour - outre du noir et du blanc - de la triade jaune / rouge / bleu, largement adoptée depuis dans lart occidental.

L'expérience, représentée sur la gravure et décrite dans le livre, consiste à comparer les tâches lumineuses formées par une lampe à une flamme et une lampe à deux flammes. D’Aguillon établit que l'éclairement de la première lampe est plus important que celui de la lampe à deux flammes placée à une distance double. Mais d'Aguillon ne formalise pas par une loi mathématique cette décroissance de l'éclairement avec la distance. C'est donc un prototype de photomètre qui n'aurait jamais été utilisé sauf par les deux génies dessinés par Rubens!

\section{Figure 1.}

Extrait Manuscrit de France Léonard de Vinci Manuscrit C folio 22 recto, circa 1490-1491.

\section{LOI EN CARRÉ INVERSE}

La loi de décroissance de léclairement avec la distance à la source lumineuse avait pourtant été établie par Johannes Kepler, quelques années auparavant, en 1604 dans son livre Astronomia pars Optica. Pour comprendre cette loi, il faut bien saisir qu'un éclairement est une densité de flux lumineux par unité de surface. Or, si dans un cône partant de la source $S$, un flux de lumière traverse une surface élémentaire à une distance $\mathrm{d}=1$, la surface traversée à une aire 4 fois plus grande à la distance $\mathrm{d}=2$, et 9 fois plus grande à la distance $\mathrm{d}=3$. La densité de flux sur une surface dénommée A sur la figure 2 diminue bien avec l'inverse de la distance au carré.

\section{Figure 2.}

Diminution de l'éclairement avec la distance: loi en carré inverse. https://fr.wikipedia.org/wiki/ Loi_en_carr\%C3\%A9_inverse\#/media/ File:Inverse_square_law.svg

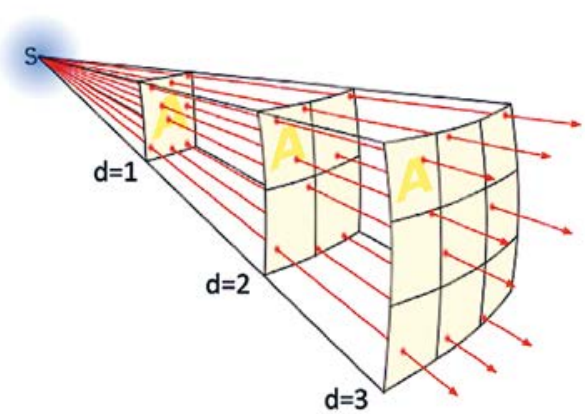

La première véritable mesure photométrique, utilisant correctement la loi en carré inverse semble avoir été faite par le scientifique Christian Huygens en 1698 pour comparer la luminosité du Soleil et celle de Syrus.

Il observe les deux astres à travers un long tube équipé d'un diaphragme réglable. Les luminosités (on dirait aujourd'hui les luminances) varient en inverse de l'ouverture du diaphragme. Mais la méthode sera critiquée trois décennies plus tard par Pierre Bouguer. La comparaison des luminosités émises par les deux astres à plusieurs heures d'intervalle ne lui paraît pas fiable [1].

\section{NOVEMBRE 1725 :}

NAISSANCE DE LA PHOTOMÉTRIE

Pierre Bouguer est de son vivant un scientifique reconnu surtout pour ses études sur la navigation: les formes des bateaux, les manœuvres, les mesures pour s'orienter en mer... Bien qu'essentiels, ses travaux en photométrie ne constituent qu'une part mineure de ses activités scientifiques. En 1729, il publie un Essai d'optique sur la gradation de la lumière qu'il complète dans les toutes dernières années de sa vie. Son essai devient un Traité d'optique et est publié à titre posthume en 1760 .

Grand expérimentateur, Pierre Bouguer pose les bases de la photométrie :

- il décrit le principe méthodologique d'égalisation deluminosité pour deux sources de lumière observées simultanément;

- il propose divers dispositifs permettant la mesure (figure 3) ;

- il utilise correctement la loi en carré inverse.

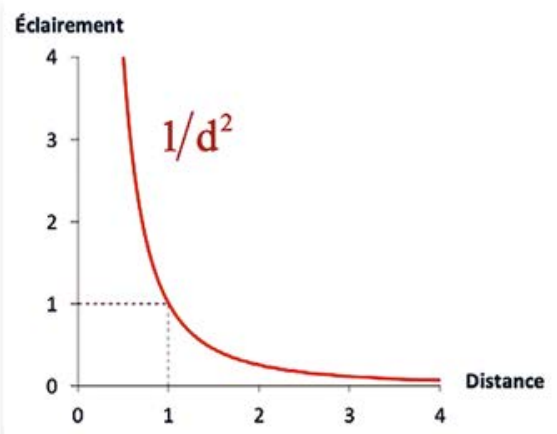


Dans son essai, Pierre Bouguer indique même la date de ce qui peut être considérée comme la naissance de la photométrie [2]. Dans la nuit du 23 novembre 1725, il compare l'éclairement produit sur une feuille par la lune et celui produit par quatre bougies. En ajustant la distance des bougies pour obtenir l'égalisation des luminosités, il estime la brillance de la lune.

\section{AMÉLIORATIONS ET DISPARITION DES PHOTOMĖTRES}

Malgré la rigueur méthodologique de Bouguer, la précision des mesures n'est pas au rendez-vous. A la fin du $\mathrm{XVIII}^{\mathrm{e}}$ siècle, Rumford propose un photomètre basé (comme sur les schémas de Léonard de Vinci !) sur la comparaison des ombres et non des éclairements directs des sources lumineuses elles-mêmes. L'œil se fatigue moins et l'observateur a plus de temps pour ajuster précisément la luminosité. Pour s'affranchir de la subjectivité des mesures, des photomètres thermométriques sont développés mais ne connaissent pas un grand succès. Peu efficaces pour la photométrie stellaire en raison de la faible luminosité des étoiles, les photomètres thermométriques sont aussi trop complexes à utiliser pour quantifier les performances des brûleurs des lampes à gaz apparues au XIX ${ }^{\mathrm{e}}$ siècle [3]. Les praticiens préfèrent le photomètre de Bunsen en raison de sa simplicité et de son faible coût. Le composant principal est une feuille blanche qu'une tâche d'huile rend translucide en son centre. La feuille est placée entre les deux sources de lumière à comparer

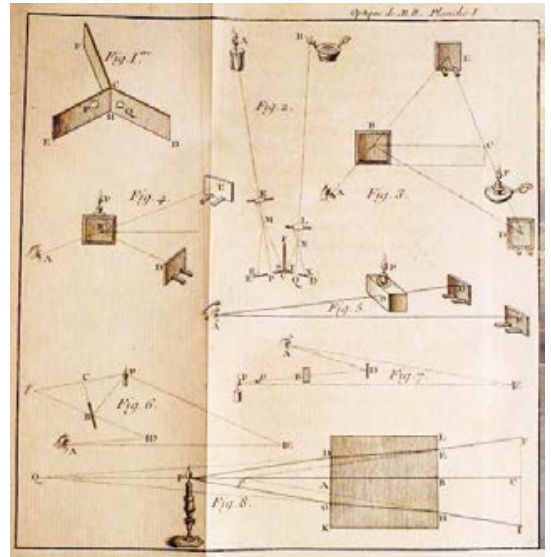

Figure 3.

Divers systèmes de mesures photométriques. Planche du Traité d'optique de Pierre Bouguer.

et l'égalisation des éclairements se produit lorsque la tâche de graisse est aussi lumineuse que le reste du papier et devient donc invisible.

Le principe d'égalisation des photomètres impose l'utilisation de sources de lumière de référence. Les «bougies », souvent différentes d'un pays à l'autre, deviennent les unités de l'intensité lumineuse. L'unification de ces unités est progressive et ce n'est qu'au milieu du $\mathrm{XX}^{\mathrm{e}}$ siècle que les «bougies » sont remplacées par la candela, une des sept unités de base du système international. Entre temps, le développement de détecteurs photoélectriques rend obsolète le principe des photomètres par égalisation visuelle. Ils sont remplacés par les luxmètres et autres luminancemètres ne faisant plus appel à la subjectivité d'un opérateur.

\section{The Swiss Army Knife of optics}

\section{HASO R-Flex2}
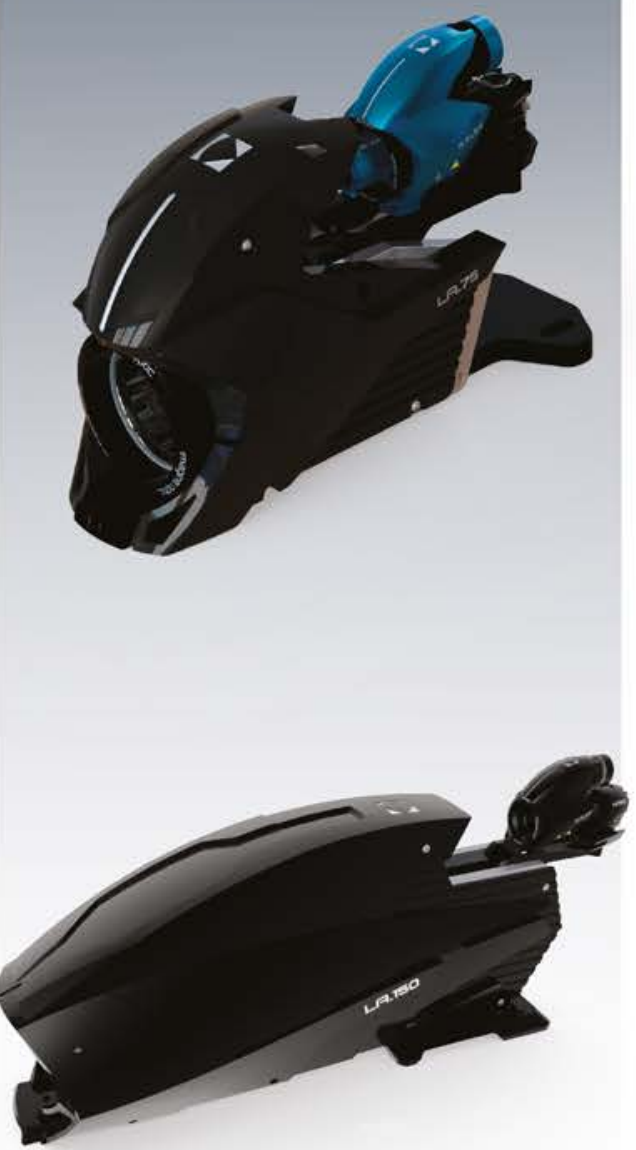

HASO R-Flex2 SWIR

\section{More on Youtube}

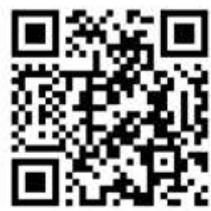

[1] J.-E. Morère, La photométrie : les sources de l'Essai d'Optique sur la gradation de la lumière de Pierre Bouguer 1729, dans Revue d'histoire des sciences et de leurs applications 18, n4, 337-384 (1965)

[2] W. E. Knowles Middleton, The beginnings of photometry, Appl. Opt. 10, 2592-2594 (1971)

[3] X. Chen, Visual Photometry in the Early $19^{\text {th }}$ Century: A "Good" Science with "Wrong" Measurements, dans : Wrong for the Right Reasons. Springer, Dordrecht, 161-183 (2005) 